PROCEEDINGS OF THE

AMERICAN MATHEMATICAL SOCIETY

Volume 137, Number 12, December 2009, Pages 4255-4257

S 0002-9939(09)10035-7

Article electronically published on August 3, 2009

\title{
LOIS DU LOGARITHME ITÉRÉ AVEC PONDÉRATIONS ADDITIVES
}

\author{
GÉRALD TENENBAUM
}

(Communicated by Richard C. Bradley)

\begin{abstract}
We provide a very short proof that natural Lindeberg type conditions on the non-negative arithmetic additive function $f$ ensure the strong law of large numbers and the law of the iterated logarithm for weighted sums $\sum_{n \leqslant N} f(n) X_{n}$ for any sequence $\left\{X_{n}\right\}_{n=1}^{\infty}$ of i.i.d. random variables.
\end{abstract}

Cette note a pour but d'étendre et de simplifier des résultats récents de BerkesWeber [1] et Fukuyama-Komatsu [3, relatifs au comportement asymptotique de sommes du type

$$
\sum_{1 \leqslant n \leqslant N} f(n) X_{n}
$$

où les $X_{n}$ sont des variables aléatoires indépendantes et de même loi et $f$ est une fonction arithmétique additive positive ou nulle.

Posons

$A_{N}=A_{N}(f):=\sum_{p^{\nu} \leqslant N} \frac{f\left(p^{\nu}\right)}{p^{\nu}}\left(1-\frac{1}{p}\right), \quad B_{N}^{2}=B_{N}(f)^{2}:=\sum_{p^{\nu} \leqslant N} \frac{f\left(p^{\nu}\right)^{2}}{p^{\nu}} \quad(N \in \mathbb{N})$,

où, ici et dans la suite, la lettre $p$ désigne un nombre premier. Les résultats de 1 , établis sous l'hypothèse que $f$ est fortement additive, positive ou nulle, et telle que 1

$$
f(p)=o\left(B_{p}\right) \quad(p \rightarrow \infty)
$$

énoncent que les relations

$$
\begin{aligned}
\lim _{N \rightarrow \infty} \frac{\sum_{n \leqslant N} f(n) X_{n}}{N A_{N}}=\mathbb{E} X_{1} \quad \text { p.s., } \\
\limsup _{N \rightarrow \infty} \frac{\sum_{n \leqslant N} f(n) X_{n}}{A_{N} \sqrt{2 N \log _{2} N}}=\left\|X_{1}\right\|_{2} \quad \text { p.s., }
\end{aligned}
$$

sont valables, dans le cas de (2), si $X_{1}$ est intégrable, et, dans le cas de (3), si $X_{1}$ est centrée et de carré intégrable. Ici et dans la suite, nous désignons par $\log _{k}$ la $k$-ième itérée de la fonction logarithme.

Received by the editors April 25, 2009, and, in revised form, April 29, 2009.

2000 Mathematics Subject Classification. Primary 60F15, 11N37; Secondary 60G50.

${ }^{1}$ Les auteurs de [1] utilisent $\sqrt{B_{n}}$ à la place de $B_{n}$. Nous nous conformons ici à l'usage traditionnel.

(C)2009 American Mathematical Society Reverts to public domain 28 years from publication 
Nous étendons les résultats précédents au cas d'une fonction additive positive ou nulle générale tout en affaiblissant significativement les conditions de validité. Toutefois, notre motivation réside également dans la production d'une preuve directe très courte, répondant ainsi à une question implicitement soulevée dans [1].

Théorème 1. Soient $\left\{X_{n}\right\}_{n=1}^{\infty}$ une suite de variables aléatoires indépendantes et de même loi, et $f$ une fonction arithmétique additive positive ou nulle vérifiant

$$
B_{N}=o\left(A_{N}\right) \quad(N \rightarrow \infty) .
$$

Alors la relation (2) a lieu dès que $X_{1}$ est intégrable. De plus, la relation (3) a lieu si $X_{1}$ est centrée et de carré intégrable, et si $f$ vérifie en outre2

$$
\sum_{p^{\nu} \leqslant N} \frac{f\left(p^{\nu}\right)^{3}}{p^{\nu}} \ll A_{N}^{3} \quad(N \rightarrow \infty) .
$$

Remarques. (i) Il est immédiat que l'hypothèse

$$
\max _{p^{\nu} \leqslant N} f\left(p^{\nu}\right)=o\left(B_{N}\right) \quad(N \rightarrow \infty),
$$

prolongeant (1), implique (4) et (5). Une fonction additive naturelle qui ne satisfait pas cette condition suffisante mais vérifie (4) et (5) est la fonction nombre de facteurs premiers, $f(n)=\Omega(n):=\sum_{p^{\nu} \| n} \nu$. Cette fonction n'étant pas fortement additive, elle ne relève pas non plus des résultats de 3 .

(ii) La condition de Lindeberg, considérée dans [3] dans le cas où $f$ est fortement additive,

$$
(\forall \varepsilon>0) \quad \sum_{\substack{p^{\nu} \leqslant N \\ f\left(p^{\nu}\right)>\varepsilon B_{N}}} \frac{f\left(p^{\nu}\right)^{2}}{p^{\nu}}=o\left(B_{N}^{2}\right)
$$

implique également (4) de façon immédiate.

Démonstration. Sous l'hypothèse (4), l'inégalité de Turén-Kubilius implique classiquement (voir par exemple le th. III.3.3 de [5]) que l'on a

$$
S_{N}:=\sum_{n \leqslant N} f(n) \sim N A_{N} \quad(N \rightarrow \infty)
$$

et

$$
T_{N}:=\sum_{n \leqslant N} f(n)^{2} \sim N A_{N}^{2} \quad(N \rightarrow \infty) .
$$

De plus, on a, uniformément lorsque $N \rightarrow \infty$,

$$
A_{M} \sim A_{N} \quad(\sqrt{N} \leqslant M \leqslant N) .
$$

Cela résulte de (4) via l'inégalité de Cauchy-Schwarz, comme indiqué dans la démonstration du résultat de 5 précédemment cité.

Ainsi que noté dans 11, la loi forte des grands nombres établie dans [4] (th. 3), permet de réduire la preuve de (2) à celle de l'estimation

$$
\sum_{S_{N} \leqslant t f(N)} 1 \ll t \quad(t \geqslant 1) .
$$

\footnotetext{
${ }^{2}$ Ici et dans la suite, le symbole de Vinogradov $a \ll b$ signifie $a=O(b)$.
} 
Or, pour $t$ assez grand, le membre de gauche de (9) n'excède pas

$$
t+\sum_{2^{j+1}>t} \sum_{2^{j} \leqslant N<2^{j+1}} \frac{2 f(N)^{2} t^{2}}{N^{2} A_{N}^{2}} \ll t+\sum_{2^{j+1}>t} \frac{t^{2}}{2^{j}} \ll t,
$$

où nous avons utilisé (6), (7) et (8). Cela établit notre première assertion.

Comme dans [1, nous utilisons ensuite le fait que, compte tenu d'estimations donnés dans [2], la seconde assertion résulte directement de la majoration

$$
\sum_{T_{N} \leqslant t f(N)^{2}} 1 \ll t \quad(t \geqslant 1)
$$

Or, désignant par $p_{j}$ des nombres premiers, on a, en vertu de (5),

$$
\begin{aligned}
\sum_{n \leqslant N} f(n)^{3} & =\sum_{p_{1}^{\nu_{1}}, p_{2}^{\nu_{2}}, p_{3}^{\nu_{3}} \leqslant N} f\left(p_{1}^{\nu_{1}}\right) f\left(p_{2}^{\nu_{2}}\right) f\left(p_{3}^{\nu_{3}}\right) \sum_{\substack{n \leqslant N \\
p_{j}^{\nu_{j}} \| n(1 \leqslant j \leqslant 3)}} 1 \\
& \ll N A_{N}^{3}+N A_{N} B_{N}^{2}+N \sum_{p^{\nu} \leqslant N} \frac{f\left(p^{\nu}\right)^{3}}{p^{\nu}} \ll N A_{N}^{3},
\end{aligned}
$$

où nous avons scindé la somme triple selon le nombre de $p_{j}^{\nu_{j}}$ distincts. Nous voyons ainsi que le membre de gauche de (10) est

$$
\ll t+\sum_{2^{j+1}>t 2^{j} \leqslant N<2^{j+1}} \frac{f(N)^{3} t^{3 / 2}}{N^{3 / 2} A_{N}^{3}} \ll t+\sum_{2^{j+1}>t} \frac{t^{3 / 2}}{2^{j / 2}} \ll t .
$$

Cela établit bien notre seconde assertion.

\section{REMERCIEMENT}

L'auteur tient à remercier l'éditeur de lui avoir signalé l'existence de l'article très récent 3 .

\section{BIBLIOGRAPHIE}

1. I. Berkes et M. Weber, A law of the iterated logarithm for arithmetic functions, Proc. Amer. Math. Soc. 135, no. 4 (2007), 1223-1232. MR2262929 (2007j:60043)

2. E. Fisher, A Skorohod representation and an invariance principle for sums of weighted i.i.d. random variables, Rocky Mountain J. Math. 22, no. 1 (1992), 169-179. MR1159950 (93e:60065)

3. K. Fukuyama et Y. Komatsu, A law of large numbers for arithmetic functions, Proc. Amer. Math. Soc. 137, no. 1 (2009), 349-352. MR2439459

4. B. Jamison, S. Orey et W. Pruitt, Convergence of weighted averages of independent random variables, Z. Wahrscheinlichkeitstheorie und Verw. Gebiete 4 (1965), 40-44. MR0182044 (31:6268)

5. G. Tenenbaum, Introduction à la théorie analytique et probabiliste des nombres, troisième édition, coll. Échelles, Paris, 2008.

Institut Élie Cartan, Université Henri Poincaré-Nancy 1, BP 239, 54506 Vandeuvre LÈes Nancy Cedex, France

E-mail address: gerald.tenenbaum@iecn.u-nancy.fr 